\title{
Development and Application of 3.7GHz LHCD system on HL-2A and Development of RF Heating system on HL-2M
}

\author{
XingYu Bai ${ }^{1, *}$, Bo Lu ${ }^{1}$, Hao Zeng ${ }^{1}$, Jun Liang ${ }^{1}$, Chao Wang ${ }^{1}$, GuoLiang Xiao ${ }^{1,3}$, He Wang ${ }^{1}$, YaLi Chen ${ }^{1}$, JieQiong Wang , \\ Kun Feng ${ }^{1}$, ShaoDong Song ${ }^{1}$, Mei Huang ${ }^{1}$, Jun Rao ${ }^{1}$, XianMing Song ${ }^{1}$, Rui Mao ${ }^{1}$, Jun Cheng ${ }^{1}$, YiPo Zhang ${ }^{1}$, DeLiang Yu ${ }^{1}$, \\ Yan Zhou ${ }^{1}$, ZhongBing Shi ${ }^{1}$, Annika Ekedahl ${ }^{2}$, Julien Hillairet ${ }^{2}$, Emmanuel Bertrand ${ }^{2}$, Lena Delpech ${ }^{2}$, Gerardo Giruzzi ${ }^{2}$, \\ Tuong Hoang ${ }^{2}$, Roland Magne ${ }^{2}$, Didier Mazon ${ }^{2}$, Yves Peysson ${ }^{2}$, XiaoLan Zou ${ }^{2}$ and the HL-2A Team ${ }^{1}$ \\ ${ }^{1}$ Southwestern Institute of Physics (SWIP), P.O. Box 432, Chengdu, China. \\ ${ }^{2}$ CEA, IRFM, F-13108 Saint-Paul-lez-Durance, France \\ ${ }^{3}$ Department of Engineering Physics, Tsinghua University, Beijing, China.
}

\begin{abstract}
The first Lower Hybrid (LH) experiments were carried out with a Passive-Active Multijunction (PAM) launcher in H-mode plasmas. The experiments were performed on the HL-2A tokamak with the new 3.7 GHz LHCD system, installed and tested by SWIP in collaboration with CEA/IRFM. The ELMs and local gas impact on LH power coupling was studied in the experiments. The coupled LH power in HL-2A was $200-500 \mathrm{~kW}$ at large gap at the first experiments and reaches $900 \mathrm{~kW}$ now in H-mode, while it reaches $1 \mathrm{MW}$ in L-mode. The LH experiments on HL-2A show that the PAM launcher is a viable concept for high performance scenarios. The LH power can be coupled at large plasma-launcher gap, and assist in triggering and sustaining H-modes. Finally, an overview of the RF heating systems for the tokamak HL-2M is given. HL-2M will dispose of a 4 MW LH system and a 8 MW ECRH system, both of which are currently under installation at SWIP.
\end{abstract}

\section{Introduction}

Lower hybrid current drive (LHCD) is a well known method of non-inductive current drive in tokamaks, which has been used successfully in tokamaks around the world since the early 1980s. A fraction of the plasma current is replaced by the non-inductively LH driven current in the tokamak by injecting LHW into one toroidal direction, which can increase the duration of the plasma discharge[1].

In view of foreseeing a LH system for the second phase of ITER [2], a relevant LH launcher design, the passive-active multijunction (PAM), has been developed [3]. The first experimental test of a PAM module was carried out in the FTU tokamak $[4,5]$. Following this, a PAM launcher was designed and constructed for Tore Supra [6], demonstrating long pulse operation at high coupled power (2.7 MW, $80 \mathrm{~s}$ ) and ITER-relevant power density [7]. So far, the PAM experiments in FTU and Tore Supra have shown excellent results of power handling and coupling at large plasma-launcher distances, making it an attractive launcher design for operation in high performance scenarios and in long pulses.

However, this has so far only been demonstrated in L-mode plasmas. Now, as a part of the $3.7 \mathrm{GHz}$ LHCD system constructed on HL-2A tokamak, the third PAM launcher is developed and applied, making it possible to carry out $\mathrm{LH}$ experiments in $\mathrm{H}$-mode plasmas by PAM antenna.

\section{LHCD system description}

The PAM launcher was designed by SWIP (Southwestern Institute of Physics) in Chengdu, in cooperation with IRFM (Institut de Recherche sur la Fusion par confinement Magnetique) at CEA. The layout of the LHCD system is depicted in Fig. 1 [8].The four klystrons are located on the ground floor and the launcher in the torus hall on the first floor. Eight transmission lines ( $\sim 20 \mathrm{~m}$ each) connect the klystrons to the launcher.

* Corresponding author: baixy@swip.ac.cn 


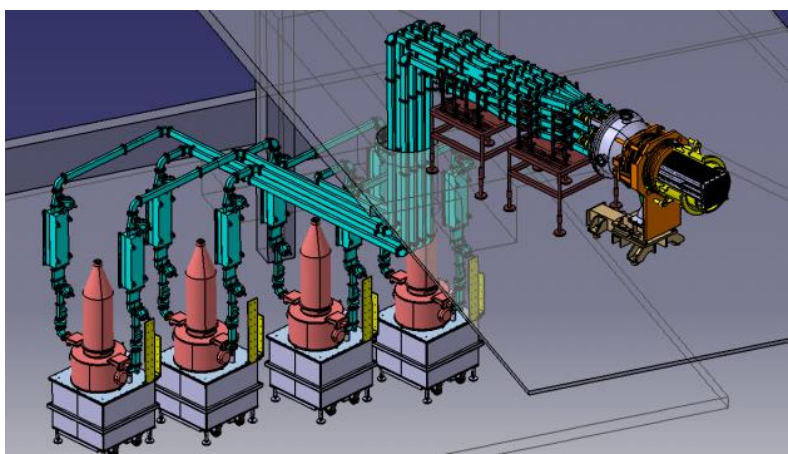

Fig. 1. Schematic layout of the $3.7 \mathrm{GHz}$ LHCD system.

\subsection{PAM launcher}

The PAM launcher consists of 16 modules, with four active and four passive grills each, mounted in four rows and four columns. One passive grill is added at the end of each toroidal row, resulting in four rows with 16 active and 17 passive grills (see photo in Fig. 2). The size parameters of the PAM mouth are width $=365 \mathrm{~mm}$; height $=325 \mathrm{~mm}$.

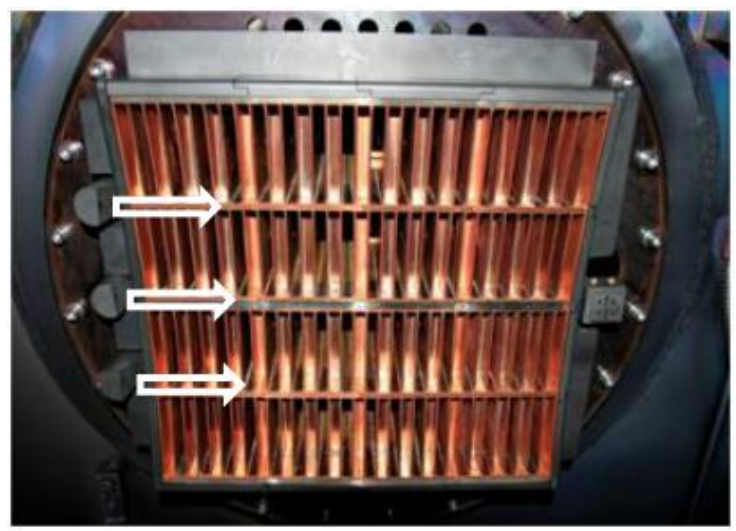

Fig. 2. Photo of the PAM launcher in HL-2A

A dedicated gas puffing system with three poloidal injection points is installed next to the launcher mouth, on the left of the launcher, signed by the arrows in Fig 2. Local gas puffing near LH launchers has been used routinely, for example in JET [9] and EAST [10], as a method to increase the local density and improve the LH coupling, in particular during $\mathrm{H}$-mode operation. A set of Langmuir probes, consisting of a total of eight probes mounted at two radial locations, is installed on the right hand side of the launcher mouth. The probes allow measuring the electron density at two radial locations and will thus also allow deducing the density and temperature decay lengths at the launcher location.

The ALOHA coupling code [11] was used for defining the design parameters for the PAM launcher. The peak parallel refractive index was chosen to $n_{/ / 0}=$ 2.75 , in order to allow accessibility of the LH wave in
HL-2A plasmas. The width and height of each grill are $8.8 \mathrm{~mm}$ and $72.14 \mathrm{~mm}$, respectively, with a wall thickness of $2.2 \mathrm{~mm}$. The depth of the passive grill is $24.5 \mathrm{~mm}$, i.e. a quarter of a wavelength. According to the thermal and electromagnetic force analysis, the launcher is made of copper to avoid local overheating in arcing and covered by stainless steel in order to protect the antenna from the twisting in plasma disruption. As the pulse duration is less than $2.0 \mathrm{~s}$, no active cooling is installed on the launcher. The launcher can be moved in the radial direction as much as $100 \mathrm{~mm}$ in order to have a good coupling in different discharge conditions. Graphite protection tiles are installed around the front of the launcher, protruding by $2 \mathrm{~mm}$ in front of the launcher mouth. ALOHA-code analysis shows that the power reflection coefficient, averaged over the 16 modules, is less than $3 \%$ when the electron density at the launcher mouth is larger than $2 \times 10^{17} \mathrm{~m}^{-3}$, the cut-off density for the $\mathrm{LH}$ wave being $\mathrm{n}_{\mathrm{co}}=1.7 \times 10^{17} \mathrm{~m}^{-3}$ at $\mathrm{f}=3.7 \mathrm{GHz}$.

\subsection{Wave source}

The PAM launcher is fed by four klystrons, giving out $500 \mathrm{~kW}$ RF power maximum via two ports each. The klystrons are powered by a high-voltage power supply (HVPS) and several auxiliary power supplies including a filament heater power supply, three electromagnet power supplies, two ion pump power supplies and an anode power supply modulator for each klystron, as suggested. But the klystrons are operated in totally a new way for the TH2013A type. A set of divider resistance is chosen to obtain the anode voltage from the cathode HVPS, replacing the anode high voltage modulator, making the HVPS system much simpler and cheaper. In this way, $500 \mathrm{~kW} / 2 \mathrm{~s}$ output power was successfully obtained from every klystron in the separately test, indicating the new way effective.

The maximum output power of the RF exciter is 10 W. Besides the four channels for power amplification, there is an additional output channel with a power level of $0 \mathrm{dBm}$ as a phase reference for the phase feedback control system, by which the $\mathrm{n}_{/ /}$spectrum can range between 2.55 and 2.95, with low reflection coefficient. The protection system can switch off the HVPS in $10 \mu \mathrm{s}$ if arcing occurs or backward power exceeds the threshold, keeping the system safe.

\subsection{Transmission lines}

The output RF power from each klystron is fed via two ports, and then divided equally to four channels by two $3-\mathrm{dB}$ power dividers. Each of the four columns of the launcher is fed by one klystron via the transmission lines, with a transmission efficiency more than $80 \%$. Since each klystron has two output arms, there are eight transmission lines in total. Eight 4-port ferrite circulators are specified near the klystrions' output windows. The 
circulator is rated to handle the full load reflection at full power over the full duration. Therefor, the experiment regime is extended with the help of the circulator. Each transmission line is about 20 meters length, based on the WR284 waveguide with a propagation mode of TE10. The transmission lines are pressurized with two bars of nitrogen in order to prevent arcing. The forward and backward power at the output windows near the klystron $(8+8=16$ channels $)$ and the input ports of the antenna $(16+16=32$ channels $)$ is measured by bidirectional couplers. As a result, the LH power mentioned in this paper refers to the pure power injected into the tokamak, reflected power subtracted.

\section{LH coupling experiments}

Prior to the experiments in H-mode plasmas, the klystrons and the PAM launcher had been commissioned in L-mode plasmas, both limiter and divertor configuration, up to $500 \mathrm{~kW}$ coupled power in $400 \mathrm{~ms}$ length pulses on HL-2A[12]. With the commissioning, the reflection coefficient is decreased much from $80 \%$ at the first experiments.

In the following experiments, coupling optimization in H-mode plasmas was carried out. Coupling effect of ELMs and local gas puffing at the antenna mouth was investigated. 200-500 kW LH power was successfully coupled during type I ELMs in H-mode plasmas triggered and sustained by Neutral Beam Injection (NBI) combined with LH waves, with a large gap (more than $10 \mathrm{~cm}$ ) between LCFS and the antenna mouth. The coupled power reached $900 \mathrm{~kW}$ in $\mathrm{H}$-mode and $1 \mathrm{MW}$ in L-mode by the optimization. The experimental results indicate that the PAM launcher is a viable concept for high performance scenarios. The LH power can be coupled at large plasma-launcher gap, and assist in triggering and sustaining $\mathrm{H}$-modes.

\subsection{Coupling effect on ELMs}

The LH coupling experiments with the PAM in H-mode were carried out in a lower single null (LSN) configuration with plasma current Ip $=160 \mathrm{kA}$, toroidal magnetic field $\mathrm{Bt}=1.4 \mathrm{~T}$ and edge safety factor $\mathrm{q}_{95}=4$. In shot \#26862, the distance from the last closed flux surface (LCFS) to the PAM launcher mouth was $11.5 \mathrm{~cm}$ at the mid-plane. Fig. 3 is the time evolution of the coupled LH power, the average RC, the $\mathrm{D} \alpha$ signal that shows the ELM behavior and the line integrated density. $\mathrm{H}$-mode is triggered $20 \mathrm{~ms}$ after the LH power is applied, when the density reaches $2.1 \times 10^{19} \mathrm{~m}^{-3} .700 \mathrm{~kW}$ of NBI power is injected between $\mathrm{t}=500 \mathrm{~ms}$ and $\mathrm{t}=1000 \mathrm{~ms}$.

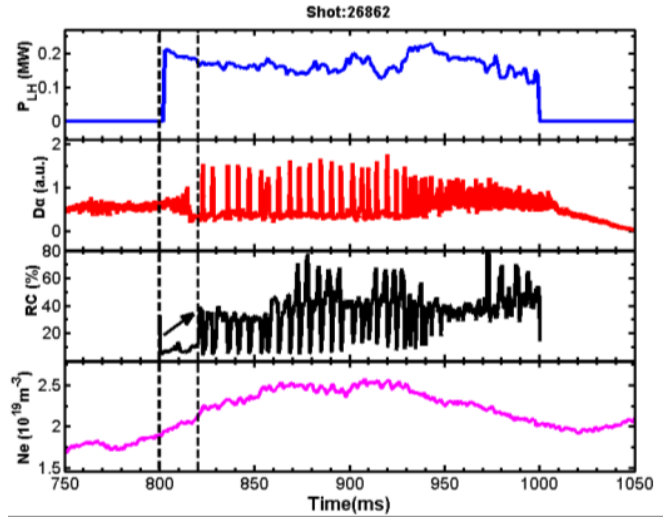

Fig. 3. LH coupling of shot \#26862.

Fig. 3 also shows the evolution of the average reflection coefficient during the ELMs. As can be expected, RC is highest during the phase between ELMs, as the density in the scrape-off-layer (SOL) is lowest in this phase. During the ELM-bursts, the electron density increases and RC decreases significantly, as expected by ALOHA modeling. RC varies between $5 \%$ and $35 \%$ during an ELM exciting. In order to show the RC behavior during an ELM more clearly, RC is plotted against the D $\alpha$ signal in Fig. 4a, while Fig. 4 b shows RC on the electron density given by Langmuir probes compared with the calculation results using ALOHA code, in the same duration. Fig. $4 \mathrm{~b}$ gives the computed $\mathrm{RC}$ for the PAM at different electron density decay lengths in front of the launcher mouth, namely $2 \mathrm{~mm}, 5$ $\mathrm{mm}$ and $10 \mathrm{~mm}$. The experimental $\mathrm{RC}$ has the same trend of density but a little higher than that of the calculation. The exact value of the density in front of the launcher, as measured by the Langmuir probes, can be quite difficult to determine. If one assumes that the density at the launcher mouth is somewhat smaller than the value given by the probe, since the launcher mouth is retracted behind its protection limiters, the experimental curve would agree better with the ALOHA code. It can be noted that in Fig. 4a, the RC behaves differently during the ELM-rise and after the ELM-crash. One plausible hypothesis is enhanced SOL ionization by the LH power during an ELM, as was investigated and modeled in JET [13]. During the ELM-crash, the SOL temperature increases and so the ionization by the $\mathrm{LH}$ power is enhanced. The ionization will increase the electron density locally in front of the powered grills, leading to a reduction in reflected power. However, this local increase in electron density may not necessarily be measured by the Langmuir probes, since they are located outside of the grill region. 

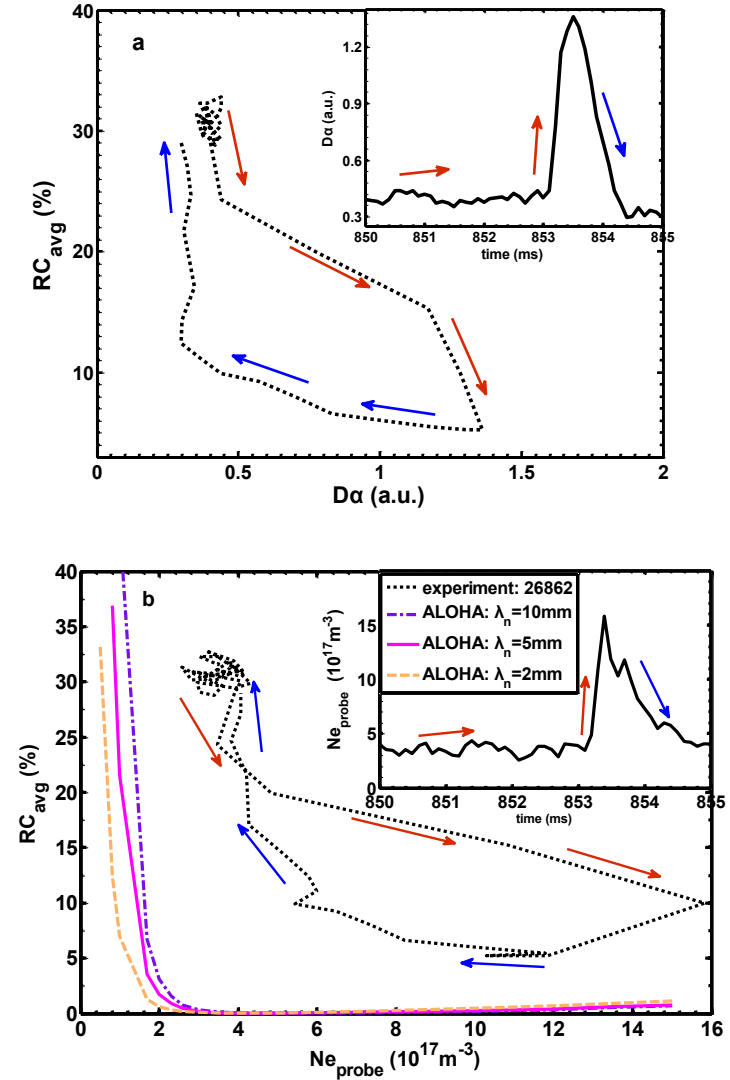

Fig. 4. $\mathrm{RC}$ versus the amplitude of the $\mathrm{D} \alpha$ signal during one ELM (a) and versus the electron density measured on Langmuir probes on the launcher compared with calculation of ALOHA code for three different density decay lengths (b).

\subsection{Coupling effect on local gas puffing}

The local gas injection was found to be crucial in this experiment for increasing the local electron density at the launcher, since the plasma-launcher gap was always very large (usually more than $10 \mathrm{~cm}$ ). The gas dosing was controlled by opening the valve during short intervals. This timing of the gas puffs is indicated in Fig. 5. The coupled LH power, RC, the D $\alpha$ signal showing the ELM behavior and the density measured by a Langmuir probe at the antenna mouth are shown in the figure. The timing of the gas puffs are indicated by dashed vertical lines.

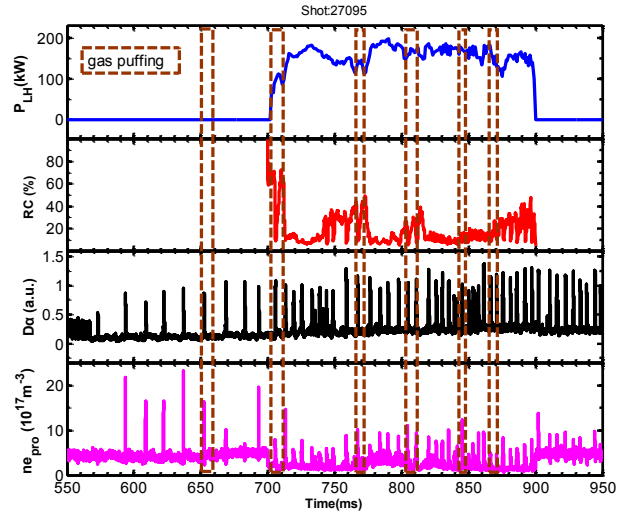

Fig. 5. LH coupling on gas injection, shot \#27095

According to Fig. 5, an increase in ELM-frequency (at around $\mathrm{t}=740 \mathrm{~ms}$ and $850 \mathrm{~ms}$ ) can be observed. The reflection coefficient decreases typically $10 \mathrm{~ms}$ after the gas puff is switched on. In this discharge, the average $\mathrm{RC}$ decreases to $8 \%$ following the gas puffs.

\subsection{Coupling optimization}

In the first coupling experiments, $200-500 \mathrm{~kW}$ LH power was coupled to H-mode plasmas via PAM antenna. Coupling optimization such as local gas puffing, plasma position control, antenna position set, and also plasma parameters changing were carried out in the experiments, decreasing $\mathrm{RC}$ to $10 \%$.

Following the first experimental campaign in 2015, a number of modifications on the LH system and on the plasma control system were carried out in order to increase the coupled LH power. A piece of shorter flexible waveguide was changed in order to reduce transmission line losses and the RF measurements at the directional couplers were improved to get better reliability of forward and reflected power measurements in each module. On the plasma control system side, the divertor coil currents could be better controlled so as to maintain a smaller plasma-launcher gap during the $\mathrm{H}$ mode phase. These modifications all had a significant effect on the LH power injection capability.

In the experiments carried out in June 2016 and May 2017, the plasma-launcher gap could be controlled at a smaller value during the $\mathrm{H}$-mode phase, allowing lower fraction of reflected power to the LH launcher. The coupled LH power has thus reached $900 \mathrm{~kW}$ in H-mode and $1 \mathrm{MW}$ in L-mode plasmas without any local gas injection [14].

One H-mode discharge with NBI $+\mathrm{LH}$ is shown in Fig. 6 (a). The H-mode is triggered when the LH power is applied, and the H-mode is then sustained throughout the duration of the LH power. Fig. 6 (b) shows that the coupled power reached $1 \mathrm{MW} / 1 \mathrm{~s}$ with really low average RC. The density was constant during the LH power injection. The RC was a little high for the first several $\mathrm{ms}(\sim 30 \%)$. This is mainly because of the plasma radial 
drift impacted by the LH power injection. Then RC maintained a low level about $7 \%$, and dropped to $4 \%$ after Electron Cyclotron Resonance Heating (ECRH) injected, indicating that the ECRH injection can benefit LH coupling. Local gas injection was not systematically used in these discharges, since the reflected power level was sufficiently low event at a smaller gap $(\sim 2.5 \mathrm{~cm})$ without any local gas puffing.
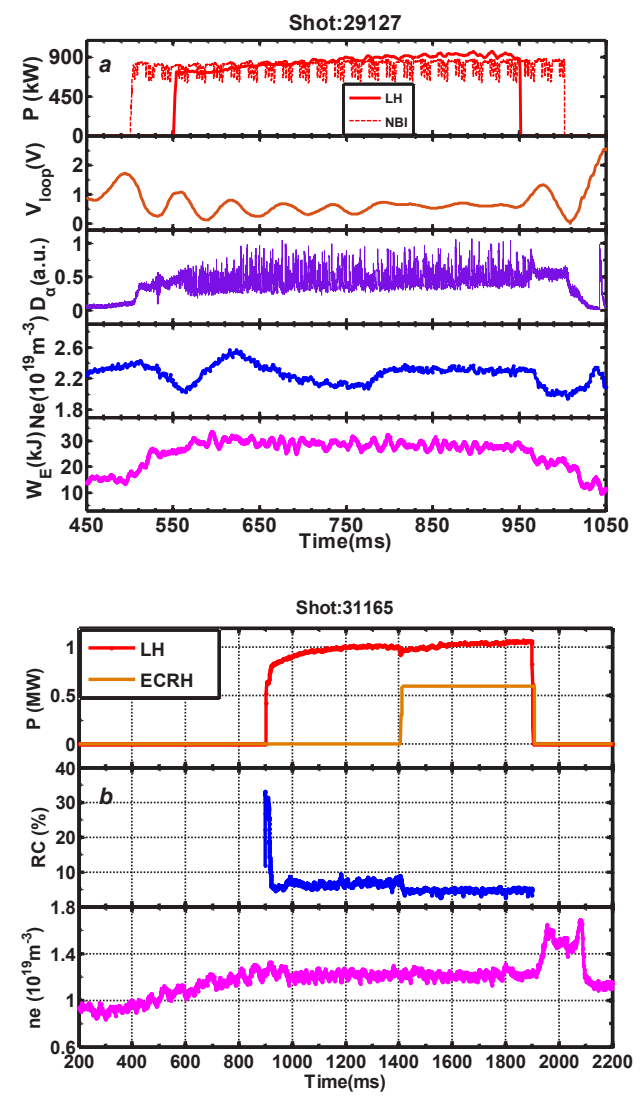

Fig. 6. 900kW LH power coupled in H-mode plasmas, shot \#29127 (a) and 1MW in L-mode, shot \# 31165 (b)

\subsection{ELMs mitigation by LH power}

The effect of LH power on the H-mode pedestal was investigated in these experiments, in particular the effect on the ELM frequency and ELM amplitude [15]. The results indicate an effect on the ELM behavior, i.e. increase in ELM frequency and decrease in ELM amplitude during LH power modulations [14], when the LH power is larger than $\sim 300 \mathrm{~kW}$. Fig. 7 is the time evolution of shot \#27141, showing ELM modification during LH injection, including coupled LH power (a), local gas puffing signal (b), ELM behavior, as seen on ion saturation current (c) and plasma radiation (d), ELM frequency (e). The mitigation effect with $\mathrm{LH}$ waves was found to be sensitive to plasma parameters and there appears to be a minimum density, ne $\sim 2.5 \times 10^{19} \mathrm{~m}^{-3}$, above which the mitigation effect occurs. An increase of the pedestal turbulence measured by Doppler reflectometry could also be observed, which suggests that an enhancement of the particle transport due to pedestal turbulence could be the cause of the ELM mitigation [14]. Similar effect was previously observed during supersonic molecular beam injection (SMBI) in HL-2A [16]. It appears that LH power injection can produce a comparable increase of the ELM frequency, as was also observed in EAST [17].

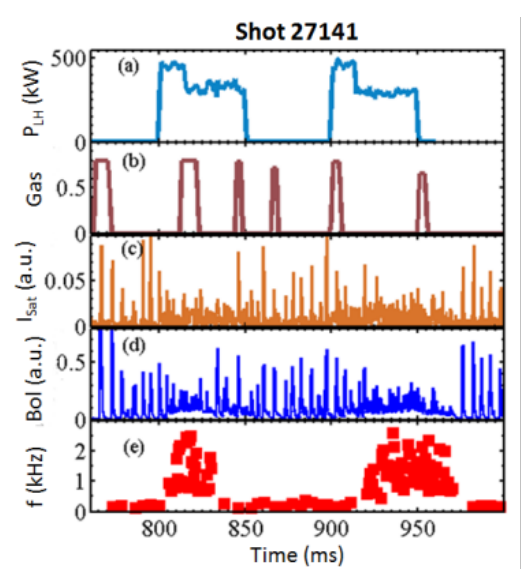

Fig. 7. ELMs mitigation by LH power injection, shot \#27141.

\section{RF heating system of HL-2M}

A new tokamak device called HL-2M is being constructed in SWIP. The RF heating system including LHCD and ECRH system are developed. HL-2M will dispose of a 4 MW LHCD system and an 8 MW ECRH system, both of which are currently under installation.

\subsection{LHCD system}

The main parameters of the LHCD system on HL-2M are $3.7 \mathrm{GHz}$ frequency, $4 \mathrm{MW}$ power and $3 \mathrm{~s}$ duration. 2 MW wave source would be constructed for the first step with a 4 MW power capacity Full Active Multijunction (FAM) antenna. For the wave source, Four TH2103C1 klystrons are ready located in the heating hall of HL-2M. All of the auxiliary equipments are also ready for the klystron operation test, which will be started at early Jun 2017. There are 8 transmission lines in the system, about $30 \mathrm{~m}$ length each. Part of the transmission components such as power combiner, absorption load and the ceramic window have been tested since the high power test bed was built in 2015. The antenna is designed in FAM structure and the peak parallel refractive index $n_{/ / 0}$ is 2.25. TE10 to TE30 mode converters are designed for the antenna to divide the power in three parts equally in ploidal direction. As a result, There are 192 active grills $(6 \times 32)$ facing to the plasma, with passive ones at each side. 


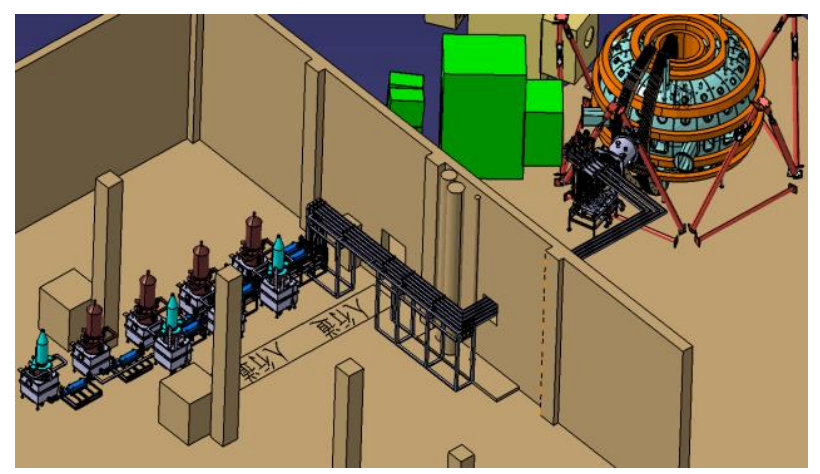

Fig. 8. LHCD system layout of HL-2M.

Fig. 8 shows the layout of the LHCD system. The smaller klystrons in the figure have not been installed yet and the bigger ones are ready.

\subsection{ECRH system}

The ECRH system takes 105/140 GHz frequency, second harmonic injection to the plasma on HL-2M. the ECRH system includes eight gyrotrons ( $1 \mathrm{MW} / 3 \mathrm{~s}$ each), two of them can be operated at dual-frequency (140 $\mathrm{GHz}$ and $105 \mathrm{GHz}$ ), other six gyrotrons operated at single-frequency $105 \mathrm{HGz}$. Fig. 9 shows the layout of the ECRH system. There are eight transmission lines, about $40 \mathrm{~m}$ length each. Corrugated circular waveguides $(\varnothing=$ $63.5 \mathrm{~mm}$ ) are applied in the transmission lines to keep the wave $\mathrm{HE}_{11}$ propagation mode. The transmission lines are pumped to $10^{-3} \mathrm{~Pa}$ level to avoid arcing. Three ports in the east of HL-2M, one equatorial port and two upper ports situated at the same vacuum segment, were allocated for ECRH launchers, corresponding to three different structure launchers, one $2 \times 3$ structure $6 \mathrm{MW}$ equatorial launcher, one $1 \times 2$ structure $2 \mathrm{MW}$ upper launcher and one $1 \times 1$ structure $1 \mathrm{MW}$ upper launcher. All of them can realize real-time feedback control in poloidal direction. For example, the response time of the equatorial launcher, which has been manufactured and tested in 2016, could reach $45 \mathrm{~ms}$ for full scan range. One can note that the total power is less than the power for launching. It is because the three antennas would not be operated at the same time. A switch will be installed in the transmission line, guiding the power to different line in different shot as wish. Up to now, six gyrotrons, four transmission lines and one antenna is ready, including the auxiliary equipments.

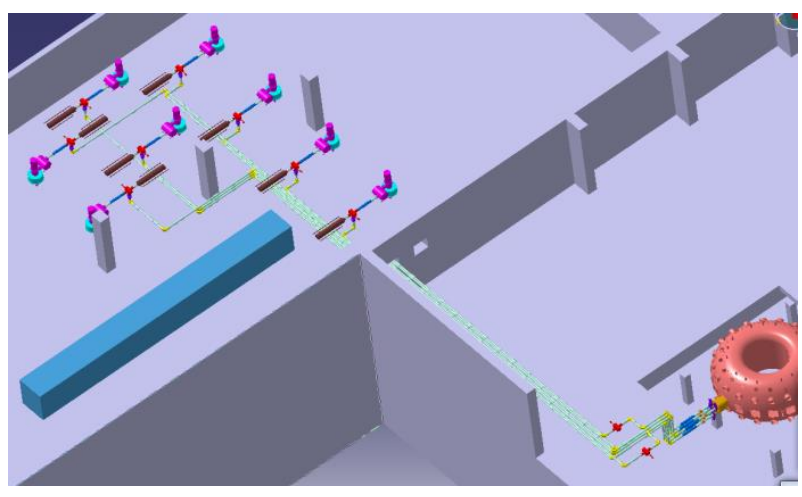

Fig. 9. ECRH system layout of HL-2M.

\section{Summary and conclusions}

A new $3.7 \mathrm{GHz}$ LHCD system was developed and applied on HL-2A, in which a new passive-active multijunction (PAM) launcher was successfully used in $\mathrm{H}$-mode experiments for the first time. The LH power was generated from 4 TH2103A klystrons, operated in a new way, and transmitted to the antenna by eight transmission lines, with a transmission efficiency more than $80 \%$. In the applying experiments, coupling optimization in $\mathrm{H}$-mode plasmas was carried out. Coupling effect of ELMs and local gas puffing at the antenna mouth was investigated. It was found that the $\mathrm{RC}$ was higher during the ELM-rise than that of after the ELM-crash in the experiments, with same trend but higher $\mathrm{RC}$ than that of calculation result via ALOHA code. The local gas puffing at the antenna mouth was found beneficial for the $\mathrm{LH}$ coupling in $\mathrm{H}$-mode plasmas with large gap. The optimization was successful since the coupled LH power reached $900 \mathrm{~kW} / 400 \mathrm{~ms}$ in $\mathrm{H}$ mode and $1 \mathrm{MW} / 1 \mathrm{~s}$ in L-mode plasmas. The LH power was found triggering and maintaining $\mathrm{H}$-mode when the plasma was heated by $800 \mathrm{~kW}$ of Neutral Beam Injection (NBI) power. It was also found that the LH power can mitigate ELMs in the H-mode plasmas. The experiments indicates that the PAM launcher is a viable concept for high performance scenarios, the LH power can be coupled at large plasma-launcher gap, and assist in triggering and sustaining $\mathrm{H}$-modes. In addition, LHCD and ECRH system of HL-2M tokamak was briefly introduced. There would be a 4 MW LHCD system and an $8 \mathrm{MW}$ ECRH system on HL-2M. Parts of the two systems have been installed and tested.

\section{Acknowledgments}

The authors gratefully acknowledge the support of the HL-2A Team at SWIP, in particular the Control \& Operation group, the Vacuum \& Gas puffing group, the Diagnostic group and also the High Voltage Power 
Supply group. This work was carried out within the framework of the Associated Laboratory in the field of fusion between CEA/IRFM and SWIP.

This work is also supported by the domestic ITER project of China (No. 2011GB104000, No. 2014GB102000), funded by Ministry of Science and Technology (MOST) and the Natural Science Foundation of China (NSFC) project (No. 11575054, No. 11261140327), funded by NSFC.

\section{References}

1. P. Bonoli, Physics of Plasmas 21 (2014) 061508

2. G.T. Hoang et al, Nucl. Fusion 49 (2009) 075001

3. Ph. Bibet et al, Nucl. Fusion 35 (1995) 1213

4. F. Mirizzi et al, Fusion Eng. Des. 74 (2005) 237

5. V. Pericoli Ridolfini et al, Nucl. Fusion 45 (2005) 1085

6. D. Guilhem et al, Fusion Eng. Des. 86 (2011) 279

7. A. Ekedahl et al., Nucl. Fusion 50 (2010) 112002

8. Lu Bo et al., Journal of the Korean Physical Society 65 (2014) 1243

9. A. Ekedahl et al., Plasma Phys. Control. Fusion 51 (2009) 044001

10. F.K. Liu et al., Nucl. Fusion 55 (2015) 123022

11. J. Hillairet et al, Nucl. Fusion 50 (2010) 125010

12. X.Y. Bai et al., Proc. 42nd EPS Conference on Plasma Physics, Lisbon (2015), paper P5.137

13. V. Petrzilka et al., Plasma Phys. Control. Fusion 54 (2012) 074005

14. X.R. Duan et al., 26th IAEA Fusion Energy Conference (2016), paper OV/4-4

15. G.L. Xiao et al. This conference poster, A-24,

16. W.W. Xiao et al., Nucl. Fusion 52 (2012) 114027

17. Y. Liang et al., Phys. Rev. Lett. 110 (2013) 235002 